\title{
Expected acceptance of the KLYPVE/K-EUSO space-based mission for the observation of ultra-high energy cosmic rays
}

\author{
Naoto Sakaki*, Shoichi Ogio \\ Graduate School of Science, Osaka City University, Osaka 558-8585, Japan \\ E-mail: sakaki@sci.osaka-cu.ac.jp
}

\section{Francesco Fenu, Mario Bertaina}

Department of Physics, University of Torino \& INFN Torino, Torino 10125, Italy

\section{Yoshiyuki Takizawa, Toshikazu Ebisuzaki}

RIKEN, 2-1 Hirosawa, Wako, Saitama 351-0198, Japan

\section{Mikhail I. Panasyuk, Pavel Klimov, Sergey Sharakin}

D.V. Skobeltsyn Institute of Nuclear Physics, M.V. Lomonosov Moscow State University, Moscow 119991, Russia

\section{for the JEM-EUSO Collaboration}

\begin{abstract}
KLYPVE/K-EUSO is a space-based observatory of ultra-high energy cosmic rays on the international space station to study anisotropy with higher statistics. The collaborative work has begun in 2013 between the original KLYPVE collaboration and the JEM-EUSO collaboration to utilize the lens technology developed for JEM-EUSO in the KLYPVE mission. The baseline optics of KLYPVE/K-EUSO consists of a mirror, a corrective Fresnel lens and a focal surface detector. The full acceptance will be about twice larger than that of Pierre Auger Observatory, and about 20\% of JEM-EUSO's. The trigger threshold energy of KLYPVE/K-EUSO was found to be possibly twice lower than that of JEM-EUSO.
\end{abstract}

The 34th International Cosmic Ray Conference,

30 July- 6 August, 2015

The Hague, The Netherlands

\footnotetext{
* Speaker.
} 


\section{Introduction}

More than 50 years have passed since the first $10^{20} \mathrm{eV}$ candidate was registered[1], but the origin of cosmic rays with energy above $50 \mathrm{EeV}$ is still a mystery. At present, two observatories for the observation of ultra-high energy cosmic rays (UHECRs) are in operation. One is the Telescope Array Project (TA) in the northern hemisphere and the other is the Pierre Auger Observatory (Auger) in the southern hemisphere. TA reported a "hot spot" (excess regions of cosmic rays above $57 \mathrm{EeV}$ in the celestial sphere) with a significance larger than 5 $\sigma$ [2]. Auger reported a dipole anisotropy and correlation with nearby Active Galactic Nuclei (AGNs)[3, 4] in the similar energy range, although the significance is smaller than the hot spot reported by TA. These results give us a hint about their origin, but the statistics are not enough to make it clear because of the very low flux above $50 \mathrm{EeV}, \sim 1$ event $/ \mathrm{km}^{2} / 100$ years. To overcome the difficulty of low statistics, several observations from a satellite orbit have been proposed, among them JEM-EUSO[5] and KLYPVE[6] projects. Both projects are space-based missions under development. The idea is to launch onto the International Space Station (ISS) a telescope to observe tracks of fluorescence and Cherenkov light in near ultra-violet band from Extensive Air Showers of UHECRs with large acceptance. Thanks to the orbit of ISS, the whole celestial sphere can be observed with a single detector with a flat exposure, which is one of the advantages for the large-scale anisotropy study. The acceptance of KLYPVE was found to be able to be improved considerably by utilizing the Fresnel lens technology developed for JEM-EUSO[6]. Collaborative work between the KLYPVE and JEM-EUSO collaborations has started in 2013[6, 7], which is called KLYPVE/K-EUSO hereafter. KLYPVE/K-EUSO is a first step to prove the ability of UHECR observation from space and of the anisotropy study with statistics higher than that of TA. Successful KLYPVE/K-EUSO mission will lead to space-based experiments with larger acceptance such as JEM-EUSO and Super-EUSO[8] to identify UHECR sources and to study their production mechanism.

\section{Baseline Optics of KLYPVE/K-EUSO}

The baseline optics for KLYPVE/K-EUSO consists of a mirror of 3.4 meter diameter, a doublesided Fresnel lens of 1.7 meter diameter and a focal surface of 1.4 meter diameter (Figure 1). The lens is made of UV-PMMA (Ultra-Violet-transparent Poly methyl methacrylate) of $10 \mathrm{~mm}$ thickness and reduces the effect of comatic aberration especially for a large field angle. Absorption loss in the lens material is less than 10\% for the wavelength longer than $320 \mathrm{~nm}$. Diffractive optics is adopted on the focal surface side of the lens to correct chromatic aberration. There are several ways to realize diffractive surfaces in general. For KLYPVE/K-EUSO, it will be realized by a Fresnel surface with groove depth of $700 \mathrm{~nm}$. The other side of the lens is a Fresnel surface with groove depth of $1 \mathrm{~mm}$. The focal surface consists of 1,900 multi-anode photomultiplier tubes with 64 pixels each with pixel size of $\sim 3 \times 3 \mathrm{~mm}^{2}$. Each photomultiplier has a BG3 band-pass filter[9] on top to select photons in the wavelength range between $290 \mathrm{~nm}$ and $430 \mathrm{~nm}$ where main lines of Nitrogen fluorescence exist. The total number of pixels are 120,000 . The data acquisition system works in photon counting mode with intelligent trigger to discriminate shower tracks[10]. The designed field of view is $\pm 14^{\circ}$ for the whole optics and $\sim 0.058^{\circ}$ for a pixel which corresponds to $0.4 \mathrm{~km}$ on the ground from the ISS altitude of $400 \mathrm{~km}$. 

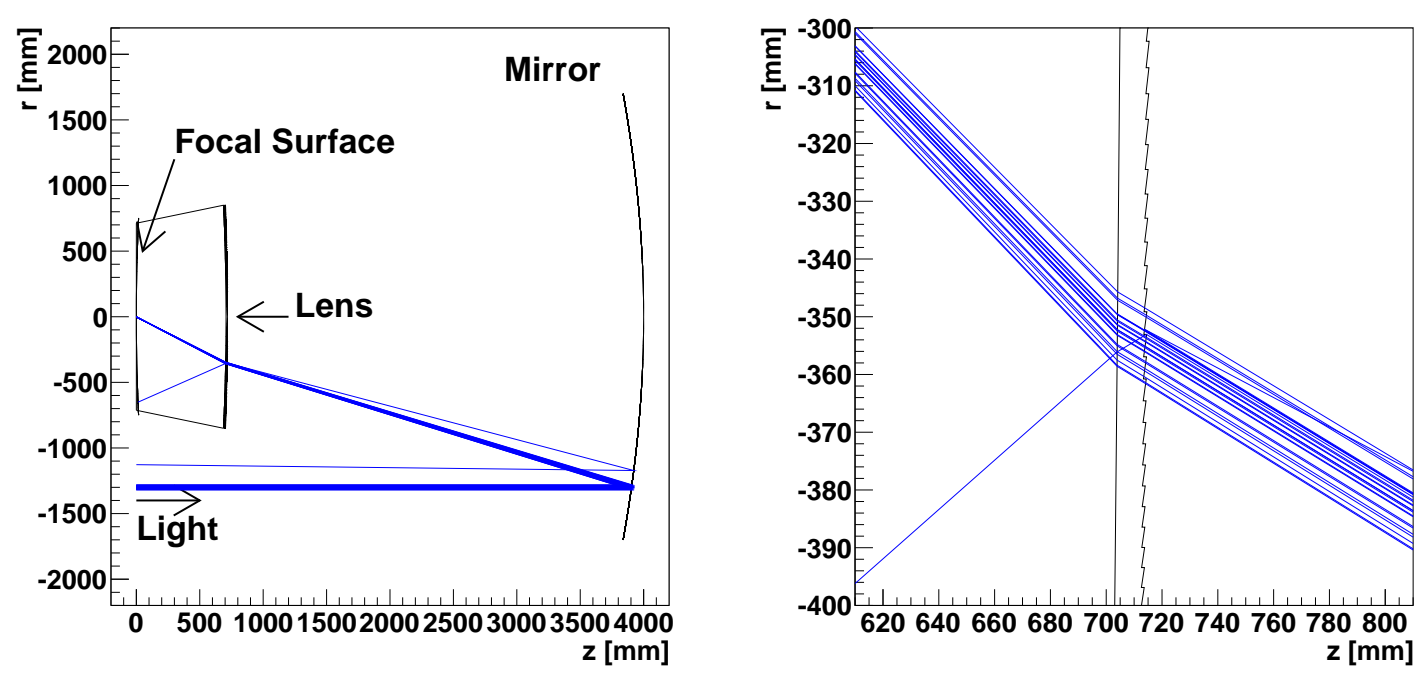

Figure 1: Schematic view of the KLYPVE/K-EUSO optics. General view of the optics is shown in the left panel and enlarged view of the lens with rays refracted and scattered by the lens in the right. Several examples of light paths are drawn with blue lines.

A dedicated raytracing code was developed to evaluate the performance of the optics. Sample rays traced by the code are shown in Figure 1. In the calculation, 90\% was used for the mirror reflectivity as a realistic number and the behavior of the rays at the diffractive surface was calculated with an optical path difference function. Figure 2 shows the shapes of the spots for three main lines of Nitrogen fluorescence (337, 357 and $391 \mathrm{~nm}$ ) and five different angles of incidence from the optical axis between 0 and 14 degrees. The photons make very complicated shapes on the focal surface depending on the wavelength and the angle of incidence. Most of the photons were concentrated at the expected position, but widely scattered photons were also observed all over the focal surface. The ratio of the scattered photons outside of the area of $20 \mathrm{~mm} \times 20 \mathrm{~mm}$ is $4 \%$ for the field angle 0 degree and increases up to $7 \%$ at 14 degrees.

In order to evaluate the performance of the optics more quantitatively, root-mean-square spot radius is plotted as a function of angle in the left panel of Figure 3. The size is comparable to the pixel size up to 14 degrees. Taking into account the tail of the point spread function outside of the pixel size, the trigger will be issued based on the signal-to-noise ratio in $3 \times 3$ pixels.

The transmittance of the optics is plotted in the right panel of Figure 3. Here we define the transmittance as the ratio of the number of photons which reach area within $3.5 \mathrm{~mm}$ radius on the focal surface to those incident onto the area within $1,700 \mathrm{~mm}$ radius at the entrance of the optics which is at $z=0$ in Figure 1. The center of the incident area was translated in the plane perpendicular to the optical axis according to the angle so that maximum number of photons reached the focal surface. In the calculation, we assumed 5\% loss due to the manufacturing error of the lens and $95 \%$ as the diffraction efficiency, which will be verified and tuned in the future by comparing with measured values of prototype optical elements. The transmittance is about $50 \%$ for 0 degree and decreases with larger angle, because more photons will miss the lens at larger angle. Larger the lens diameter becomes, more photons will be obscured by the lens and less photons will miss 
the lens at large angle. We have decided 1.7 meter as an optimum diameter of the lens taking into account the both effect. The budget of the photon loss in the optics for $357 \mathrm{~nm}$ and 0 degree for example is as follows. $25 \%$ of incident photons will be blocked naturally by the focal surface lens structure. $10 \%$ will be lost at the mirror and about $13 \%$ will be scattered or reflected by the Fresnel lens structure. The overall transmittance was estimated as $47 \%$ after taking into account other smaller factors such as absorption in the material, diffractive efficiency and the loss due to the manufacturing error of lens.
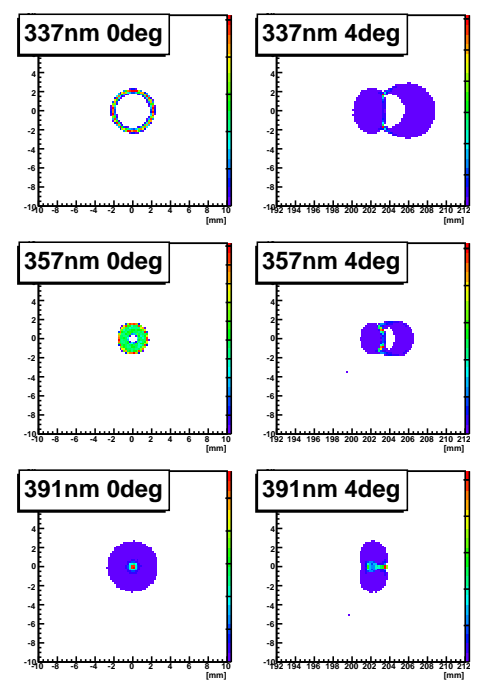
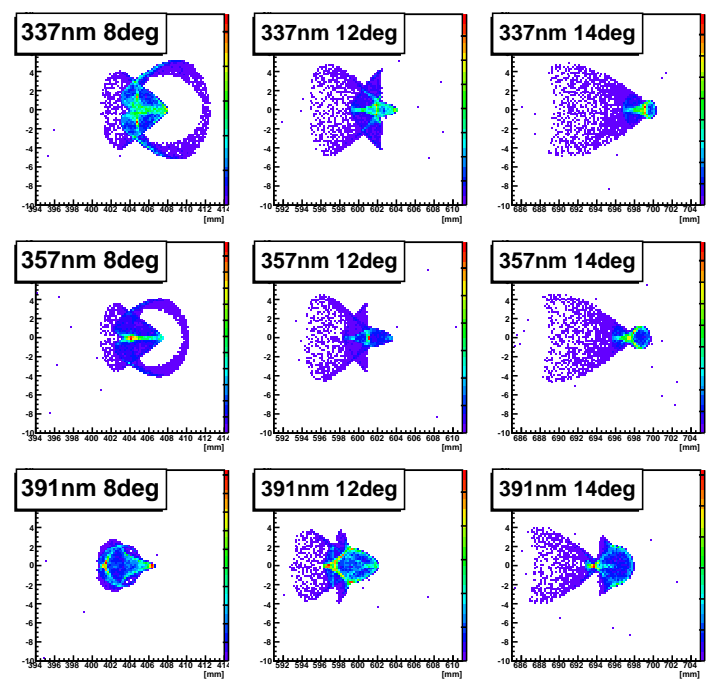

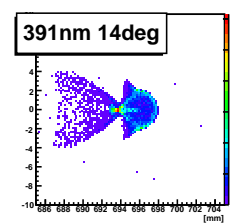

Figure 2: Spot diagram of the KLYPVE/K-EUSO optics. The panels in each row show the diagrams for the wavelength 337, 357, and $391 \mathrm{~nm}$, respectively and those in each column show for the light with incident angle from the optical axis, $0,4,8,12$ and 14 degrees. The size of each frame is $20 \mathrm{~mm} \times 20 \mathrm{~mm}$. The arrived number of photons are more intense as the color changes from blue to red.
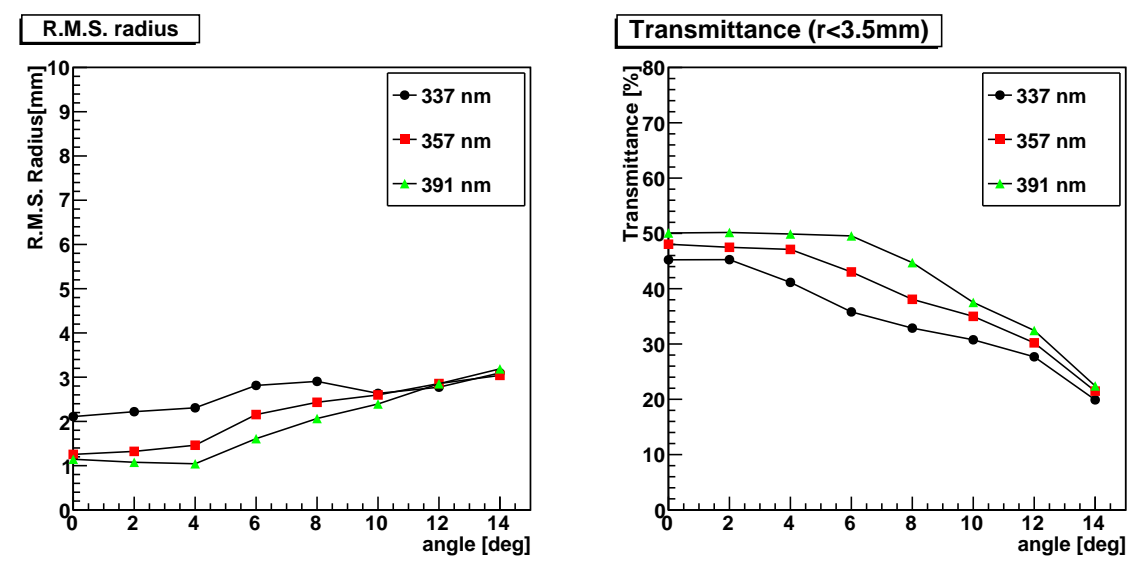

Figure 3: Root-Mean-Square (RMS) spot radius (left) and optics efficiency (right) of the KLYPVE/KEUSO optics as a function of incident light angle for the wavelength 337 (dots), 357 (squares) and $391 \mathrm{~nm}$ (triangles). 


\section{Acceptance of KLYPVE/K-EUSO for UHECR observation}

The raytracing code of the KLYPVE/K-EUSO has been implemented into the Euso Simulation and Analysis Framework (ESAF)[11] to evaluate the performance of the cosmic ray observation. ESAF adopted a modular structure, so that user can choose one of the implemented models in each part. In this paper, we used a parametrized shower generation according to the Greisen-IlinaLinsley (GIL) function[10, 12], fluorescence production following the Nagano's result[13], photon propagation based on LOWTRAN[14], the raytracing code for the KLYPVE/K-EUSO optics of this work and the electronics described in Ref.[10]. The background photon rate was assumed as 500 photons $/ \mathrm{m}^{2} \mathrm{sr} n s$ which we assume as a typical number above a dark part of the earth[10].
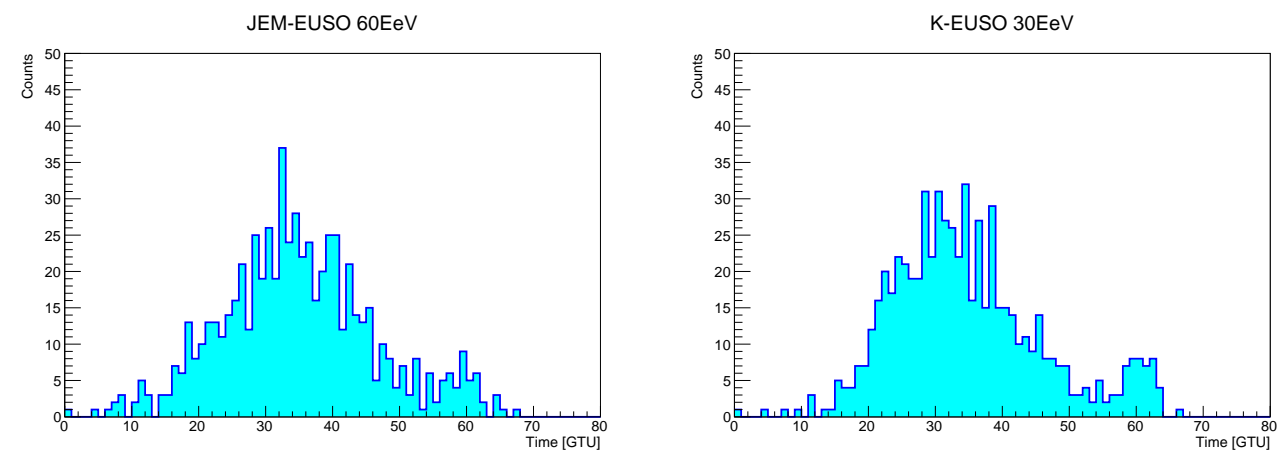

Figure 4: Samples of shower development curves observed by JEM-EUSO (left) and K-EUSO (right). Detected number of photo-electrons from a shower is plotted as a function of Gate Time Unit (GTU) which is $2.5 \mu \mathrm{s}$. Proton primary of $60 \mathrm{EeV}$ for the JEM-EUSO observation and $30 \mathrm{EeV}$ for the K-EUSO observation were injected into atmosphere. Only signals from the extensive air showers are plotted.

In Figure 4, two samples of shower longitudinal developments observed by JEM-EUSO and KLYPVE/K-EUSO are shown. The number of photo-electrons will be counted every Gate Time Unit (GTU, 2.5 $\mu \mathrm{s})$ in the electronics. Proton primary at $60 \mathrm{EeV}$ landing at the center of the field of view with zenith angle of 60 degrees was simulated for the JEM-EUSO observation, while proton at $30 \mathrm{EeV}$ with the same geometry as for the JEM-EUSO case was simulated for KLYPVE/KEUSO. As can be seen from the figure, almost same number of photo-electrons are detected for both observations. This means that the threshold energy of KLYPVE/K-EUSO will be lower than that of JEM-EUSO and the reconstruction error will be similar for $30 \mathrm{EeV}$ event by KLYPVE/KEUSO and for $60 \mathrm{EeV}$ event by JEM-EUSO[16, 17]. More detailed study on reconstruction is in progress and the result will be reported in other papers. A nominal background rate on orbit at the altitude of $400 \mathrm{~km}$ was assumed as $500 \mathrm{photons} / \mathrm{m}^{2} \cdot \mathrm{sr}$ as in Ref.[10]. This number corresponds to the detected background rate, 0.68 counts $/ \mathrm{pixel} / \mu \mathrm{s}$, after taking into account the KLYPVE/KEUSO detector response.

Since KLYPVE/K-EUSO observes UHECR only in dark nights, annual exposure will be better to describe its expected performance than acceptance. The annual exposure for triggering KLYPVE/K-EUSO was estimated as a function of energy with the following function:

$$
<\text { Annual exposure }>=A(E) \cdot \eta_{0} \cdot \kappa_{C} \cdot\left(1-f_{\mathrm{loc}}\right) \cdot(1[\mathrm{yr}]),
$$


where $A(E)$ is the trigger acceptance of KLYPVE/K-EUSO in the case of the nominal background rate, $\eta_{0}$ is the reference duty cycle due to high background rate by the sun light and the moon light, $\kappa_{C}$ is the observation efficiency where there is no cloud or extensive air showers are observable above cloud, $f_{\text {loc }}$ is the ratio of too bright area to observe cosmic rays where a big city exists or lightning happens. We chose $\eta_{0}=20 \%, \kappa_{C}=72 \%$ and $f_{\text {loc }}=10 \%$ as in Ref.[10]. The annual trigger exposure increases with energy and becomes constant above $\sim 3 \times 10^{19} \mathrm{eV}$ (Figure 5). The maximum exposure can be determined by the field of view for space-based experiments and will reach $1.2 \times 10^{4} \mathrm{~km}^{2} \cdot \mathrm{sr} \cdot \mathrm{yr} / \mathrm{yr}$ in case of nadir observation by KLYPVE/K-EUSO. This number agrees well with a simple estimation using the ratio of the field of views of KLYPVE/K-EUSO to of JEM-EUSO. It is only $\sim 1 / 5$ of the JEM-EUSO's annual exposure, but about twice of the Pierre Auger Observatory's. It should be noted that the exposure is comparable or above the JEMEUSO's at energy smaller than $2 \times 10^{19} \mathrm{eV}$ owing to the larger optics area. It is a good feature for a space-based mission of the first generation. At the first stage, the results of ground-based experiments like Auger, TA can be confirmed with the space-based mission. And then at the next stage, the instrument will be tilted for the observation at the highest energy end with high statistics.

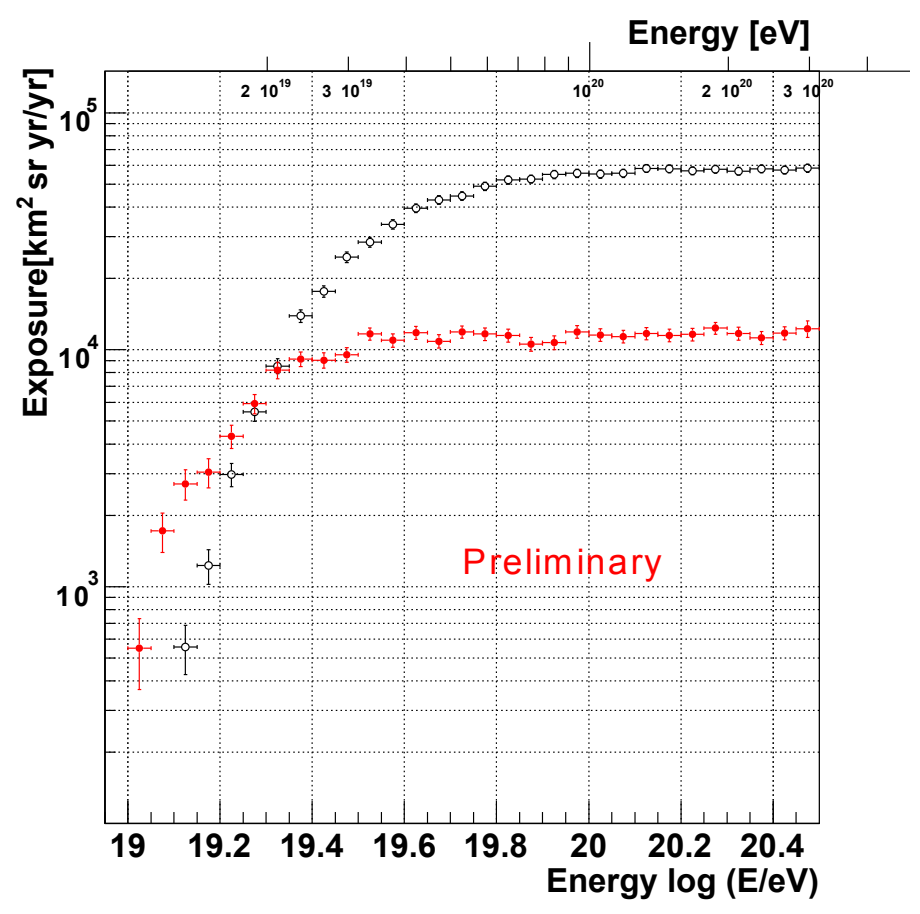

Figure 5: Annual trigger exposure of KLYPVE/K-EUSO (red solid circles) compared with JEM-EUSO's (black open circles)[10]. See the text for detailed conditions.

The integral number of cosmic rays triggering KLYPVE/K-EUSO observing towards the nadir during the mission period (6 years) is shown in Figure 6. The power law spectrum with smooth suppression based on the observation at the Pierre Auger Observatory, which was reported at the 33rd International Cosmic Ray Conference, was used to estimate the number[15]. About 1,000 cosmic rays will trigger KLYPVE/K-EUSO above $\log E>19.5$ during the mission period. Considering the flux reported by the Auger collaboration is lower than that by TA within systematic 
error, this is a conservative estimate.

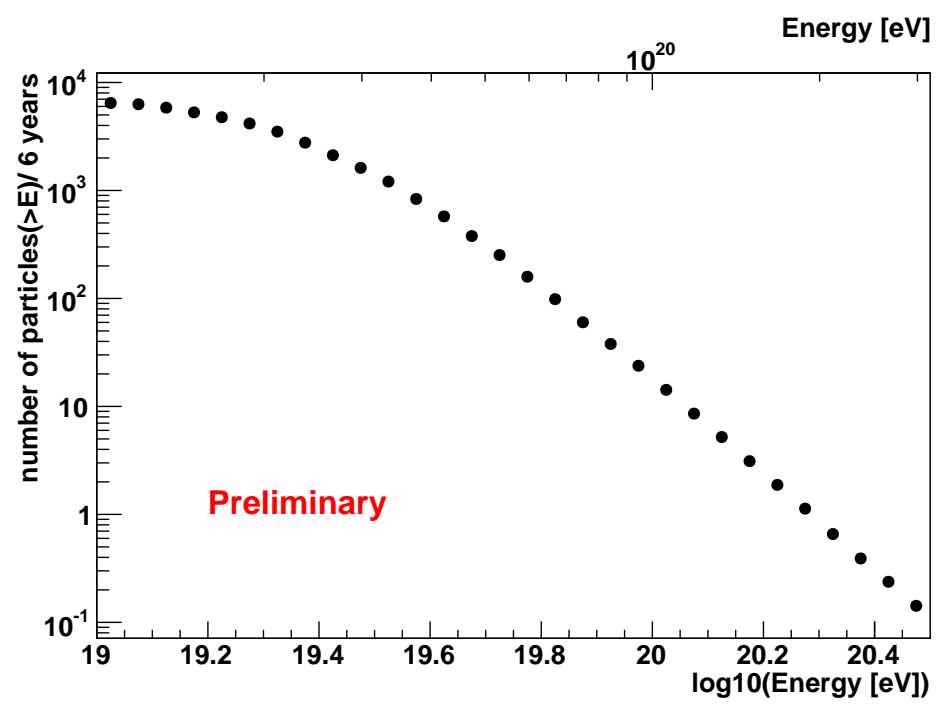

Figure 6: Integral number of triggering cosmic rays during the mission period (6 years) of KLYPVE/KEUSO observing towards the nadir. The energy spectrum reported by the Pierre Auger collaboration at ICRC2013 was used as input[15].

\section{Conclusions}

KLYPVE/K-EUSO will be a UHECR observatory on ISS in the near future. Its optics consists of a mirror, a corrective Fresnel lens and a focal surface. In this paper, a dedicated raytracing code has been developed to study the performance. The spot radius on the focal surface was found to be $\sim 3 \mathrm{~mm}$ or smaller for all over the field of view, $\pm 14^{\circ}$. The optics efficiency was $\sim 50 \%$ for photons parallel to the optical axis. The performance for cosmic ray observation was evaluated with the full simulation framework for EUSO, ESAF, with the raytracing code implemented. The annual trigger exposure will be $1.2 \times 10^{4} \mathrm{~km}^{2} \cdot \mathrm{sr} \cdot \mathrm{yr} / \mathrm{yr}$. The threshold will be lower than that of JEMEUSO and the annual exposure at $2 \times 10^{19} \mathrm{eV}$ will be comparable. This feature will be good for the establishment of space-based experiments in connection with the ground-based experiments. As soon as the spectrum is confirmed, the instrument will be tilted to increase the exposure to observe UHECRs at the high energy end with higher statistics. KLYPVE/K-EUSO will open the era of UHECR observation from space. It will be followed by the experiments with larger exposure like JEM-EUSO, Super-EUSO to study "Particle Astronomy".

Acknowledgment: This work was partially supported by Basic Science Interdisciplinary Research Projects of RIKEN and JSPS KAKENHI Grant (22340063, 23340081, and 24244042), by the Italian Ministry of Foreign Affairs, General Direction for the Cultural Promotion and Cooperation, by the 'Helmholtz Alliance for Astroparticle Physics HAP' funded by the Initiative and Networking Fund of the Helmholtz Association, Germany, and by Slovak Academy of Sciences MVTS JEM-EUSO as well as VEGA grant agency project 2/0076/13. Russia is supported by the Russian Foundation for Basic Research Grant No 13-02-12175-ofi-m. The Spanish Consortium involved in the JEM-EUSO Space Mission is funded by MICINN \& MINECO under the Space Program projects: AYA200906037-E/AYA, AYA-ESP2010-19082, AYA-ESP2011-29489-C03, AYA-ESP2012-39115-C03, AYA-ESP2013-47816- 
C4, MINECO/FEDER-UNAH13-4E-2741, CSD2009-00064 (Consolider MULTIDARK) and by Comunidad de Madrid (CAM) under projects S2009/ESP-1496 \& S2013/ICE-2822.

\section{References}

[1] J. Linsley, "Evidence for a primary cosmic-ray particle with energy $10^{20} \mathrm{eV}$ ", Phys. Rev. Lett. 10 (1963) 146-148.

[2] R. U. Abbasi et al., "Indications of intermediate-scale anisotropy of cosmic rays with energy greater than $57 \mathrm{Eev}$ in the northern sky measured with the surface detector of the telescope array experiment", The Astrophys. J. Lett. 790 (2014) L21.

[3] The Pierre Auger Collaboration, "Correlation of the highest-energy cosmic rays with nearby extragalactic objects", Science 318 (2008) 938-943.

[4] The Pierre Auger Collaboration, "Update on the correlation of the highest energy cosmic rays with nearby extragalactic matter", Astropart. Phys. 34 (2010) 314-326.

[5] P. Picozza et al. (JEM-EUSO collaboration), “The JEM-EUSO program”, Proc. 34th Int'l Cosmic Ray Conf. (The Hague) (2015) PoS(ICRC2015)618.

[6] P.I. Panasyuk et al. (JEM-EUSO collaboration), "Ultra high energy cosmic ray detector KLYPVE on board the Russian Segment of the ISS”, Proc. 34th Int'l Cosmic Ray Conf. (The Hague) (2015) PoS(ICRC2015)669.

[7] F. Kajino et al. (JEM-EUSO collaboration), “K-EUSO: An improved optical system for KLYPVE Ultra-High Energy cosmic ray space telescope”, Proc. 34th Int'l Cosmic Ray Conf. (The Hague) (2015) PoS(ICRC2015)634.

[8] A. Petrolini, "Ultra-high energy cosmic particles studies from space: Super-EUSO, a possible next-generation experiment", Nucl. Inst. Methods in Phys. Res. A 630 131-135.

[9] BG3 filter, http://www.schott.com/advanced_optics/english/download / schott-bandpass-bg3-dec-2014-en.pdf, SCHOTT AG.

[10] J.H. Adams Jr. et al. (JEM-EUSO Collaboration), "An evaluation of the exposure in nadir observation of the JEM-EUSO mission”, Astropart. Phys. 44 (2013) 76-90.

[11] C. Berat et al., "Full simulation of space-based extensive air showers detectors with ESAF", Astropart. Phys. 33 (2010) 221-247.

[12] N.P. Ilina et al., "CHERENKOV RADIATION AND PARAMETERS OF EXTENSIVE AIR SHOWERS”, Sov. J. Nucl. Phys. 55 (1992) 1540-1547.

[13] M. Nagano et al., "New measurement on photon yields from air and the application to the energy estimation of primary cosmic rays", Astropart. Phys. 22 (2004) 235-248.

[14] F.X. Kneizys et al., "User's Guide to LOWTRAN 7”, FGL-TR-0177, U.S. Air Force Geophysics Laboratory, Hanscom (1988).

[15] A. Schulz et al. for the Pierre Auger Collaboration, "The measurement of the energy spectrum of cosmic rays above $3 \times 10^{17} \mathrm{eV}$ with the Pierre Auger Observatory”, Proc. 33rd Int'l Cosmic Ray Conf. (Rio de Janeiro), (2013) \#0769 [arXiv:1307.5059].

[16] A. Guzmán et al. (JEM-EUSO Collaboration), "JEM-EUSO observational capabilities for different UHE primaries”, Proc. 34th Int'l Cosmic Ray Conf. (The Hague) (2015) PoS(ICRC2015)600.

[17] F. Fenu et al. (JEM-EUSO collaboration), "The JEM-EUSO energy and Xmax reconstruction performances”, Proc. 34th Int'l Cosmic Ray Conf. (The Hague) (2015) PoS(ICRC2015)604. 